Ethan D. Hoag

\author{
AVCO-EVERETT RESEARCH LABORATORY \\ a division of \\ AVCO CORPORATION \\ Everett, Massachusetts
}

Contract No. NAS 8-5279

June 1965

prepared for

GEORGE C. MARSHALL SPACE FLIGHT CENTER NATIONAL AERONAUTICS AND SPACE ADMINISTRATION Huntsville, Alabama 
ABSTRACT

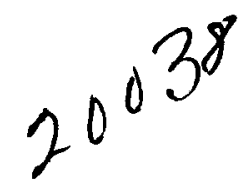

Measurements of the magnetic anisotropy of critical current in samples wide enough for use in large magnets reveal a degradation effect in strip superconductors. The effect is manifested as a truncation or "chopping off" of the peaks of the anisotropy curves of the se samples. With narrow samples the anisotropy curves (average critical current density plotted as a function of magnetic field direction) normally display a well-defined peak centered about the direction parallel to the rolling plane of the strip. With wide samples, however, this peak appears progressively more truncated as the width is increased, and the truncation level (i.e., maximum current density) is roughly inversely proportional to the width, so that the total current tends to remain constant, independent of width. For $\mathrm{Nb}-25 \% \mathrm{Zr}$ strip $0.051 \mathrm{~mm}$ thick, this limiting current is approximately $125 \mathrm{~A}$ at a field of $4 \mathrm{~Wb} / \mathrm{m}^{2}$ for widths between 1 and $10 \mathrm{~mm}$. $1.32-\mathrm{mm}$ wide samples display little or no truncation and have maximum current densitites of $1.8 \times 10^{5} \mathrm{~A} / \mathrm{cm}^{2}$, whereas $10-\mathrm{mm}$-wide samples show severe truncation and have maximum current densities of $2.6 \times 10^{4} \mathrm{~A} / \mathrm{cm}^{2}$. Q P 1 th 


\section{Critical Current Degradation in Wide Samples of High-Field Strip Superconductors}

\section{by}

\section{Ethan D. Hoag}

Measurements of the magnetic anisotropy of critical current in wide wide samples of $\mathrm{Nb}-\mathrm{Zr}$ strip superconductors have revealed a degradation effect not previously reported in the literature.

Several reports have appeared on anisotropy effects in superconductors. 1, 9 These studies, done on very narrow samples, indicate with little doubt that the anisotropy is due to basic material properties (possibly to strains introduced during rolling) rather than to sample geometry. Our measurements when taken on samples of comparable size confirm these results.

However, on wide samples with cross sections suitable for practical magnet construction, the peaks of the anisotropy curve (normally centered about the direction parallel to the rolling plane) become "chopped off" or truncated.

Our data are presented in the form of anisotropy curves for various sample widths. In the se curves the average critical current density (total critical current divided by cross-sectional area of sample) is plotted as a function of the orientation angle at constant field magnitude. The orientation angle $\theta$ is defined in Fig. 1. All data were taken with the current flowing parallel to the rolling direction and all samples were sheared from "as-rolled" $\mathrm{Nb}-25 \% \mathrm{Zr}$ strip $0.051 \mathrm{~mm}$ thick. 


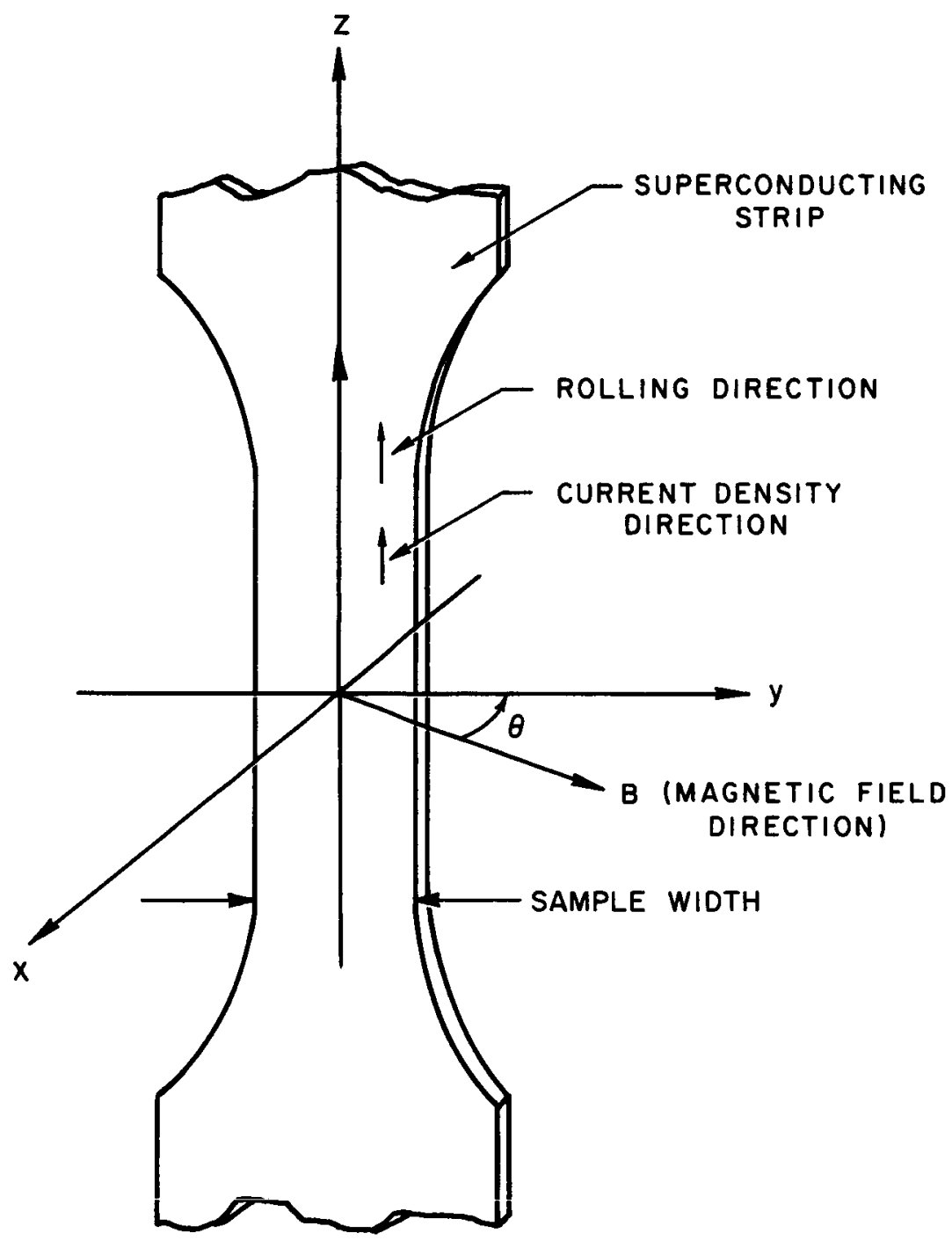

Fig. 1 Definition of Orientation Angle, $\theta$ 
Two Methods were used for energizing the strip, direct energization from an external power supply and inductive energization using a dc transformèr technique in which the sample formed a closed one-turn secondary of a transformer. With direct energization a microvoltmeter placed across the test section was used to detect the onset of the normal state. With inductive energization the normal state was detected by the decay of the sample current. In both cases the sample was heavily shunted with copper to provide an alternate current path when the sample quenched. Both methods yielded essentially the same results.

The magnetic field was obtained from a Helmholtz-type stationary superconducting magnet. Changes in orientation were effected by rotating the sample.

Ample precautions were taken to assure that the truncation observed was not due to faulty contacts or other spurious causes.

Typical results are shown in Fig. 2 where samples of four different widths, all at a field of $4 \mathrm{~Wb} / \mathrm{m}^{2}$, are compared. The $1.32-\mathrm{mm}$ sample displays a well-defined peak which is probably due to basic properties of the material. Curves for wider samples conform generally to the basic envelope but are truncated more and more as the width is increased.

The peak current in each case is approximately inversely proportional to the width so that the total current tends to remain independent of width as shown in Table I. 


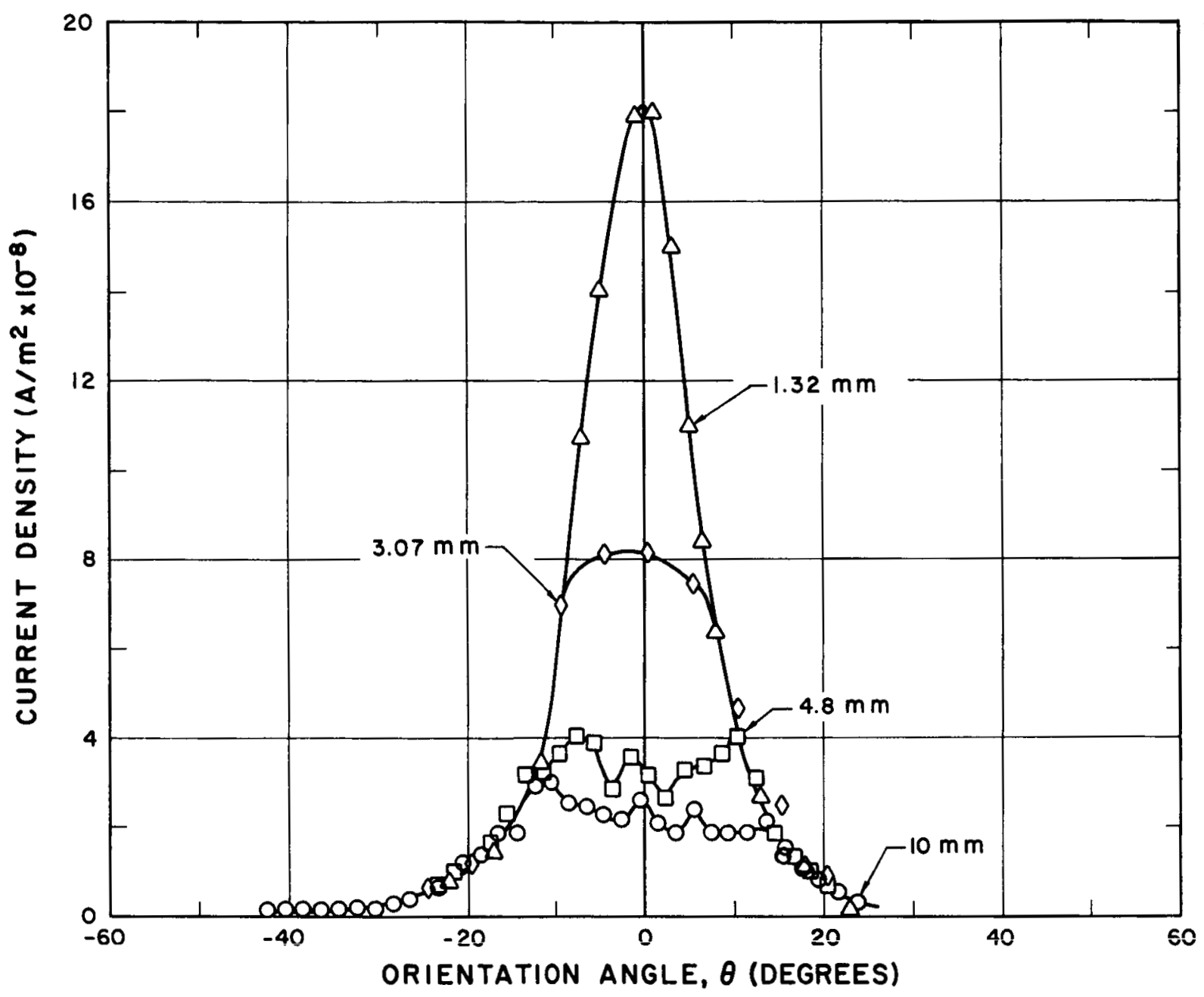

Fig. 2 The Effect of Sample Width on Anisotropy 


\section{TABLE I}

Total Current vs. Strip Width for Field Parallel to Strip Plane $(\theta=0)$.

\section{Strip Width (mm)}

1.32

3.07

4.80

10.0
Total Current (A)

80

This behavior is similar in some respects to the well-known degradation effect in superconducting magnets where the critical current of a large mass of wire in close proximity is lower than that of short sample. Here, wide samples of superconducting strip have lower critical current densities than small samples of the same material. To the extent that a wide strip can be looked upon as several narrow strips in close proximity (as far as the transport current is concerned), this is a similar effect. The reasons for the well-known magnet degradation effect, though not yet well understood are believed to stem from the "flux jump" behavior of superconductors. 10,12

To the extent that this degradation in wide strips stems from the same causes, further study of the phenomena may shed light on the nature of flux jumps and their effect on the stability of superconducting materials. 


\section{REFERENCES}

1. LeBlanc, M.A.R. and Little, W. A., Proc. 7th Int. Conf. Low Temp. Physics, 1960. (University of Toronto Press, Toronto, 1961) p 363

2. Chandrasekhar, B.S., Walker, M.S., Riemersma, H. and Werner, F.E., Proc. 8th Int. Conf. Low Temp. Physics, 1962. (Butterworths, Washington, 1963) p 345

3. Hake, R. R., Berlincourt, T.G. and Leslie, D.H., IBM J. 6 (1962) 119

4. Berlincourt, T.G., Hake, R.R. and Leslie, D.H., Phys. Rev. Ltrs. 6 (1961) 671

5. Hake, R.R., Leslie, D.H. and Berlincourt, T.G., Phys. Rev. 127 (1962) 170

6. Walker, M.S. and Fraur, M. J., Superconductors. (Interscience, N.Y., 1962) p 99

7. Tedmon, C.S., Jr., Rose, R. M. and Wulff, J., Metallurgy of Advanced Electronics Materials. (Interscience, N. Y., 1962) p 89

8. Kramer, D. and Rhodes, C.G., Metals 16 (1962) 90

9. Heise, B. H. , Linde Company, Tonawanda, N. Y. (unpublished)

10. Lubell, M.S. and Mallick, C. T., Applied Phys. Letters $\underline{4}$ (1964) 206

11. Lubell, M.S., Chandrasekhar, B.S. and Mallick, G.T., Applied Phys. Letters $\underline{4}$ (1963) 79

12. Riemersma, H., Hulm, J.K. and Chandrasekhar, B.S., Advances in Cryogenic Engineering (Volume 9, 1963) p 329 